
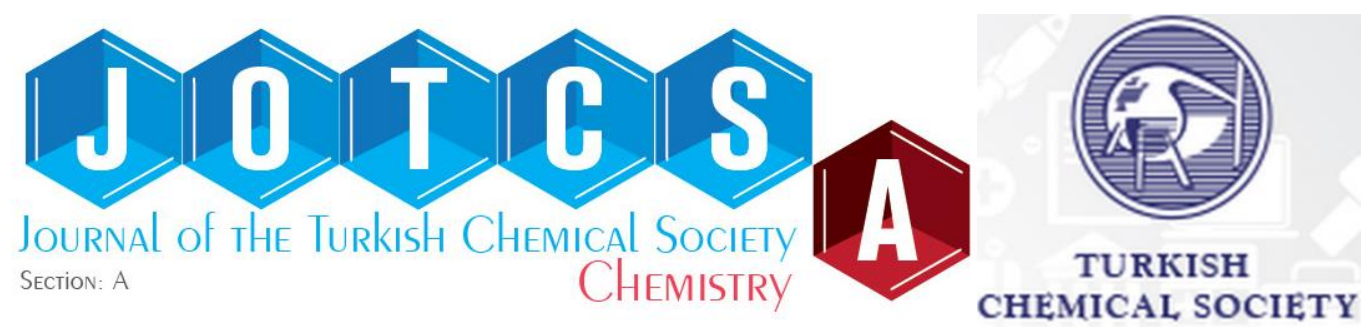

\title{
THE LATEST ADVANCEMENTS IN THE ACYLATION REACTIONS VIA CROSS- DEHYDROGENATIVE COUPLING AND/OR METAL CATALYSTS
}

\author{
Soykan Agar ${ }^{1 * i D}$, Omer T. Gunkara² \\ ${ }^{1}$ Department of Chemistry, Faculty of Science and Arts, Istanbul Technical University, Maslak, \\ Istanbul 34469, Turkey; \\ ${ }^{2}$ Department of Chemistry, Faculty of Science and Arts, Yildiz Technical University, Davutpasa \\ Campus, Istanbul, Turkey.
}

\begin{abstract}
There are quite many examples in the scientific literature regarding the acylation reactions, especially the metal-catalyzed acylation reactions, metal-free acylation reactions, metalcatalyzed acylation via cross-dehydrogenative coupling (CDC) reactions and metal-free acylation via cross-dehydrogenative coupling $(C D C)$ reactions. In this review paper, the most important examples of these domains were brought together and their mechanisms were exhibited in a clear, chronological format. Following these, the best example study towards green chemistry with a metal-free and high-yielding route was mentioned and discussed to demonstrate what has achieved in this field regarding the new acylation reaction mechanisms using the advantages of crossdehydrogenative coupling (CDC) reactions. The most prominent studies regarding these domains have been examined thoroughly and the latest progress in this field was explained in detail.
\end{abstract}

Keywords: Acylation, Cross-Dehydrogenative coupling (CDC) reactions, C-C bond formation, C-H bond activation, Metal Catalysts, Metal-free Acylation.

Submitted: September 15, 2017. Accepted: December 25, 2017

Cite this: Agar S, Gunkara O. THE LATEST ADVANCEMENTS IN THE ACYLATION REACTIONS VIA CROSS-DEHYDROGENATIVE COUPLING AND/OR METAL CATALYSTS. JOTCSA. 2018;5(1):247-68.

DOI: http://dx.doi.org/10.18596/jotcsa.338292.

*Corresponding author. E-mail: sagar11@alm.ku.edu.tr 


\section{INTRODUCTION}

Without a doubt, synthetic chemists always aim developing novel chemical reaction pathways at all times while trying to improve the mildness of reaction conditions. Such a goal is necessary to maximize the energy and product efficiency, product selectivity, chemical pathway simplicity, and environmental health and safety. Depending on this, carbon-carbon bond formation is known to be the central part of many chemical syntheses, and discoveries where these types of carbon-carbon bond formation reactions have the potential to improve overall synthetic efficiency. The acylation of aromatic compounds possesses quite a significant shelf in the library of organic chemistry. The acyl variations of aromatic compounds are widely used in the fields of drug designs, pharmaceuticals and as the building blocks in the polymer chemistry (1-3).

In order to get some environmentally benign results in the field of organic synthesis, a brand new concept of cross-coupling reaction, cross-dehydrogenative coupling, was discovered. Under the conditions of oxidative medium, from the reaction of two $\mathrm{C}-\mathrm{H}$ bonds, form directly varying $\mathrm{C}-\mathrm{C}$ bonds. Such discoveries within this cross-dehydrogenative coupling field lead us to the direct and efficient formation of $\mathrm{C}-\mathrm{C}$ bonds. Therefore, the main advantage of this issue when we lean onto the mechanistic details of CDC is that this area has the capability to become one of the most important foundations of green chemistry due to the possibility of the usage of mild medium conditions as well as serving as an auxiliary field for the technological application processes of aromatic organic syntheses; especially if there would be more studies concerning acylation reactions via metal free coupling reactions within mild conditions.

$\mathrm{CDC}$ reactions are mainly used to form bonds between $\mathrm{sp}^{3}-\mathrm{sp}^{3}, \mathrm{sp}^{3}-\mathrm{sp}^{2}, \mathrm{sp}^{3}-\mathrm{sp}, \mathrm{sp}^{2}-\mathrm{sp}^{2}$ (including Heck-type reaction), $\mathrm{sp}^{2}$-sp (Sonogashira type) and $\mathrm{sp}$-sp (Glaser reaction) carbon atoms and also $C\left(\mathrm{sp}^{3}\right)-\mathrm{N}$. Due to these plethora of varying bond formations that CDC reactions possess, the CDC definition consists of a group of reactions where the mechanism and reactivity varies and differs dramatically depending on the substrate. These above mentioned couplings can be roughly divided into four groups including CDC via Heck-type mechanism, direct arylation, CDC via ionic intermediates and CDC via radical intermediates (4).

The first group, CDC via Heck-type mechanism should be emphasized since it is a significant matter where this type mechanism (5) is a member of typical $\mathrm{sp}^{2}-\mathrm{sp}^{2} \mathrm{CDC}$ reactions including alkenealkene, arene-alkene and arene-benzoquinone coupling; and also an $\mathrm{sp}^{3}-\mathrm{sp}^{2}$ type; and a formal $\mathrm{sp}^{3}$ $\mathrm{sp}^{2}$ type concerning enolate-alkene, allyl-arene coupling. As it can be seen from the name of CDC via Heck-type mechanism, the mechanism is very similar to Heck Reaction itself. Even though the fact that the same reactivity may be achieved with transition metals such as Rh(II), Ru(III) and 
Agar and Gunkara, JOTCSA. 2018; 5(1): 247-268.

RESEARCH ARTICLE

$\operatorname{Ir}(\mathrm{I})$ complexes, Palladium (6) salts are generally chosen as the catalysts. The arene ring undergoes electrophilic palladation with an assistance of a ligand or achieved via a base mediated $\mathrm{C}-\mathrm{H}$ insertion, along with a $\mathrm{Pd}$ (II) catalyst to generate arylpalladium intermediate. Subsequent carbopalladation of olefin forms the alkylpalladium complex that further proceeds through syn- $\beta-\mathrm{H}$ elimination to yield styrenyl product and $\mathrm{Pd}(0)$. The last step is the oxidation of $\mathrm{Pd}(0)$ to $\mathrm{Pd}(\mathrm{II})$ to complete the catalytic cycle. In an alternative proposed mechanism, the Pd(II) catalyst gets used to form coordination with the olefin which enhances its electrophilicity and tendency to undergo nucleophilic addition via the electron rich aromatic rings. Such catalytic systems in the CDC via Heck-type mechanism are not usually too complex as one might think of, since they require no or solely a simple ligand as the following examples; phosphines, N-protected amino acids or sulfoxides. Any organic or inorganic base can also be utilized on top of that. In the cases where the formation of more than one product is possible, the regio-selectivity is normally achieved by the introduction of a directing group onto the aromatic ring whereas the pyridyl-based groups are the most extensively used ones to direct transition-metal-catalyzed $\mathrm{C}-\mathrm{H}$ activation reactions, both ortho- and meta- (7).

The second group of CDC reactions, the direct arylation, also known as 2-fold $\mathrm{C}-\mathrm{H}$ activation, is used to form bonds between two arenes (8). The strategy of direct arylation is an alternative route to such very well-known and widely used methods as Suzuki, Stille, Negishi, Hiyama, Kumada crosscouplings which require independent synthesis and isolation of aryl halide or pseudohalide and arylmetal starting materials.

Unlike the first two mechanisms, the third group, CDC via ionic intermediates can be promoted by various transition metals involving $\mathrm{Pd}, \mathrm{Ru}, \mathrm{Zr}, \mathrm{Ni}, \mathrm{Cu}, \mathrm{Fe}, \mathrm{Co}$ and the list goes on and on whereas the last three are the most widely used ones. Although some exceptions are known, the ionic mechanism predominantly relies on the single electron transfer (SET) pathway (9). In its mechanism, C-C/N bond formation occurs between the electrophilic carbon species (carbocations) and carbon- or nitrogen-based nucleophiles such as amine, amide, carbanion, enamine, heteroaromatic structures.

The final group of CDC reactions, CDC via radical intermediates, is very similar to the one described above (the ionic one), except the fact that no ionic intermediates get formed. The newly generated carbon centered radical, directly reacts with another active specie such as a carbocation, carboanion, C-C multiple bond, forming further a second radical which later on gets oxidized either by an oxidant (10) or transition metal (11) yielding the desired product. The radical CDC is exclusively promoted by first row transition metal complexes in the periodic table such as $\mathrm{Cu}, \mathrm{Fe}$, $\mathrm{Mn}$ (in some cases vanadium oxo-species) via single electron transfer type of a pathway. Selective 
$\mathrm{C}-\mathrm{H}$ bond oxidation of a radical can be accomplished with phenols, electron-rich arenes, benzylic compounds and $\mathrm{C}\left(\mathrm{sp}^{3}\right)-\mathrm{H}$ bond $\mathrm{a}-$ to carbonyl or heteroatom ( $\mathrm{O}$ or $\mathrm{N}$ ) structures.

These all sub-categories of CDC reactions state one important message that CDC is a promising approach to minimize the byproduct formation and reduce the total number of steps of the organic syntheses (12-26). Acylation reactions are commonly used in these aforementioned mechanisms of CDC reactions, and since they take an important place along with the CDC reactions, in this review, the latest advancements of acylation reactions and their relationship with CDC reactions were examined and explained as expressed in the following studies.

\section{The Metal Catalyzed Acylation and Coupling Reactions}

$\mathrm{Li}$ and co-workers has quite an important paper published in 2010 regarding the study of copper catalyzed intramolecular $\mathrm{C}-\mathrm{H}$ oxidation/acylation of formyl- $N$-arylformamides (27). In this study, indoline-2,3-dione was synthesized with the use of a copper catalysts by acylation. Indoline-2,3diones were known to be the building blocks that are widely used. Until 2010, there were only two other synthetic routes for indoline-2,3-diones. However, with the addition of this new route, found by Li et al., now it gained a third significant organic synthetic pathway (Scheme 1).

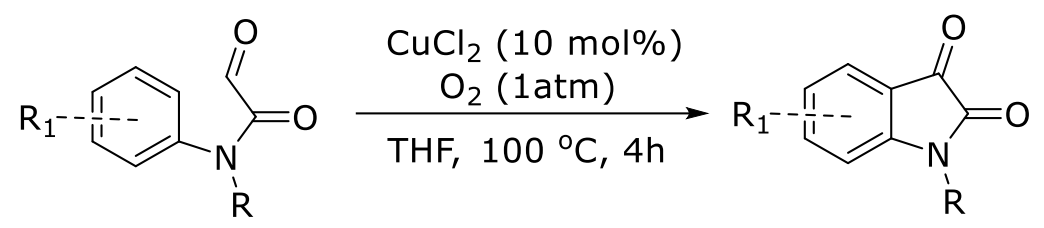

Scheme 1: The cyclization of phenylacetamide derivative with copper(II) chloride via Friedel Crafts acylation.

In the first step, $\mathrm{Cu}(\mathrm{I})$ gets oxidized to its second state. Then, $\mathrm{Cu}$ (II) forms a coordination complex with the aldehyde carbon and amide nitrogen and intermediate (I) gets synthesized. The $\mathrm{sp}^{2}$ arene carbon couples to $\mathrm{Cu}$ (III) to yield intermediate (II), following the reductive elimination of intermediate (II) yielding the product and $\mathrm{Cu}$ (I) species. The control reaction was also studied in this paper in order to exhibit whether the mechanism is free radical or not. In the existence of radical terminating agents such as TEMPO and 1,1-diphenylethylene, the product was not affected since, the mechanism does not depend on any radical. Therefore, any possibility regarding that this is a free radical mechanism can be ruled out (Scheme 2 ). 


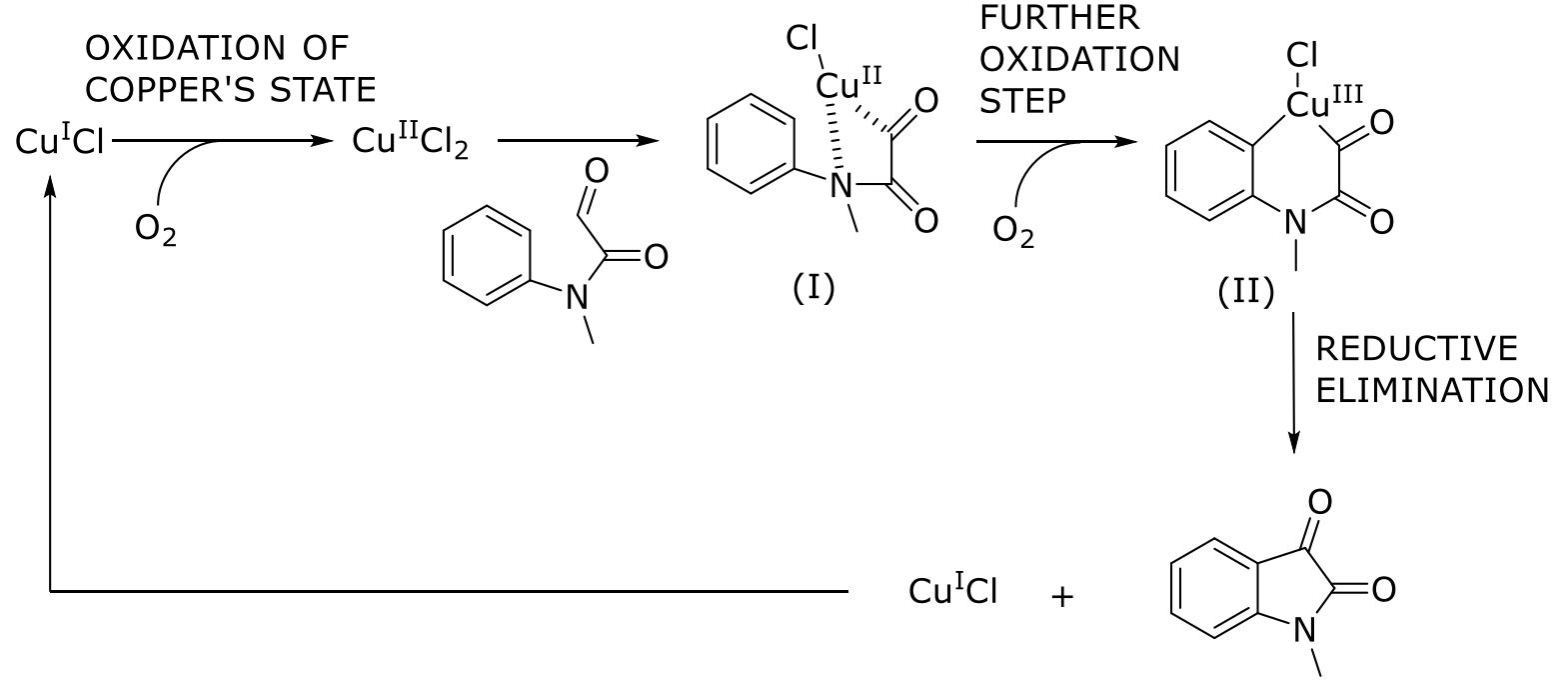

Scheme 2: The proposed mechanism of the cyclization of phenylacetamide derivative with copper(II) chloride via Friedel Crafts acylation.

In 2012, Zhu and co-workers published a study concerning Au(III)-catalyzed coupling reactions between alcohols and $\mathrm{N}$-heterocycles via $\mathrm{C}-\mathrm{H}$ bond activation. The scientific literature gained a new methodology regarding the acylation of $N$-heterocyclic compounds (28) (Scheme 3).

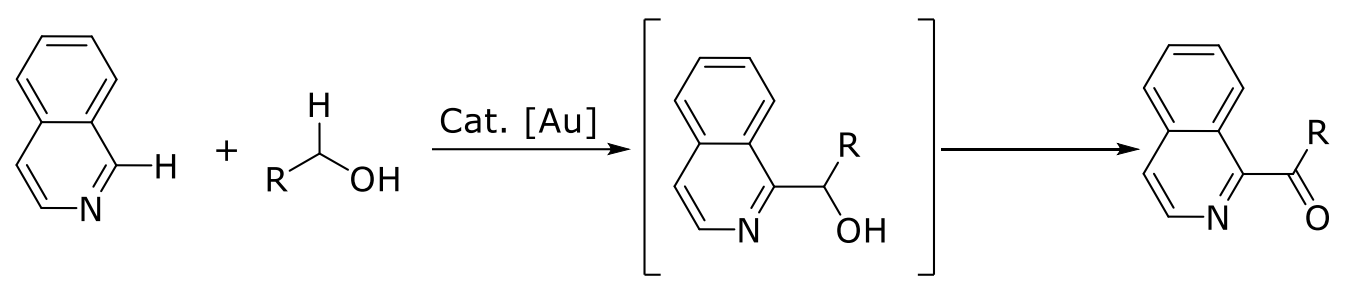

Scheme 3: Au-catalyzed dehydrogenative coupling between alcohols and isoquinoline.

In the first step, a tert-butoxide and hydroxyl radicals get formed under heat. With the hydrogen atom abstraction on the alpha position of ethanol, free radical intermediate (I) occurs. Then, this intermediate (I) reacts with the gold activated heterocyclic aromatic compound to yield intermediate (II). Intermediate (II) gets attacked by intermediate (I) to further yield intermediate (III) which is a metal complex of the heterocyclic aromatic compound. Following that, intermediate (III) gets rearomatized by the radical initiator tert-butoxide to yield the alcohol intermediate (IV). Then this alcohol intermediate (IV) is oxidized to the desired acylation product via Au/TBHP.

In the test reactions, it was observed that instead of the gold catalyst when some other metal catalysts were used, no product or some trace amounts of products were able to be obtained. This proves the necessity of the gold catalyst in the mechanism of this pathway (Scheme 4). 


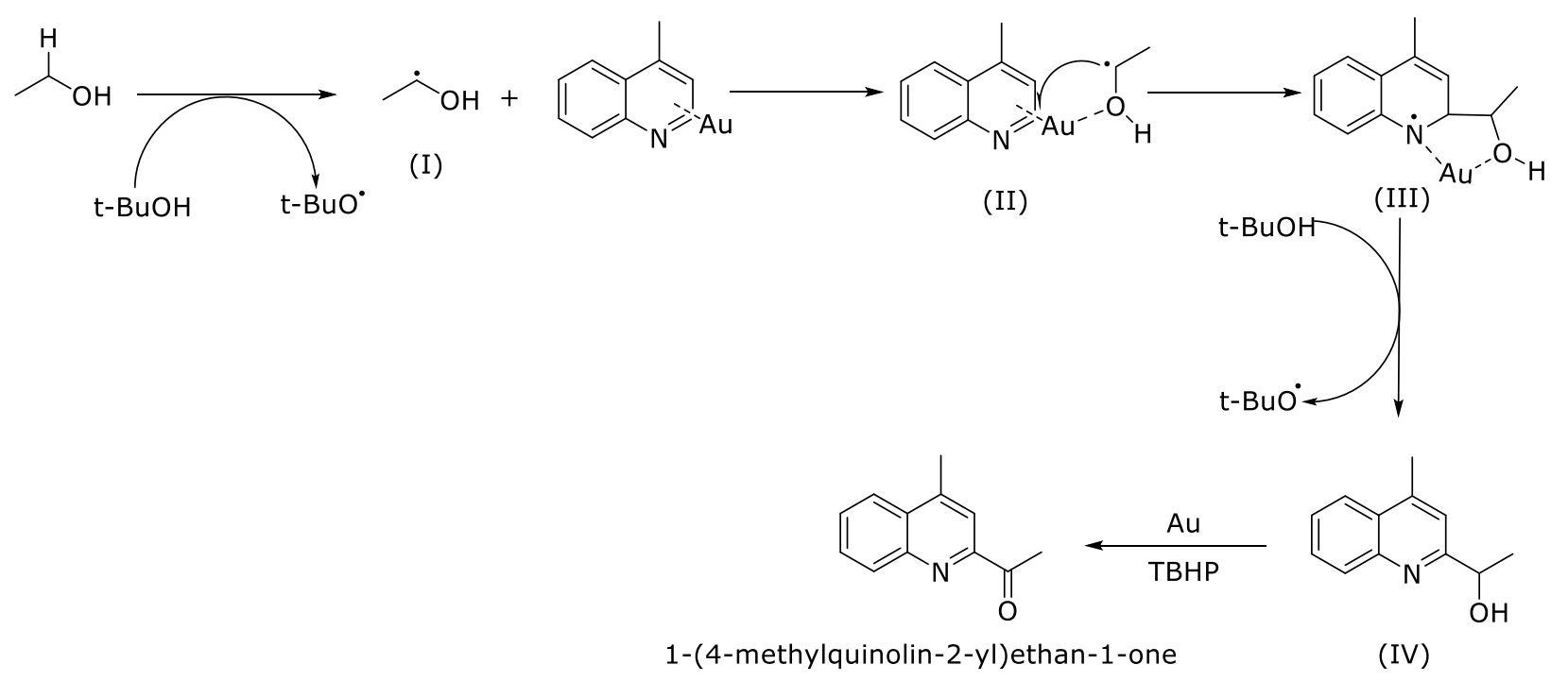

Scheme 4: The proposed mechanism of the Au-catalyzed dehydrogenative coupling reaction.

As it is well known, in the recent years, transition metal catalyzed decarboxylative cross coupling $\mathrm{C}-\mathrm{C}$ bond formations have gained significant importance. Qi and co-workers in 2014, exhibited the silver-catalyzed decarboxylative acylation of arylglyoxylic acids (29). The result that they achieved in their study is quite an efficient pathway in the synthesis of non-symmetric diaryl ketones. In such a transformation reaction, alpha-oxocarboxylate was coupled with arylboronic acid via the corresponding silver(I) carbonate within the medium to give high yields and high functional group compatibility in aprotic polar solvents (Scheme 5).

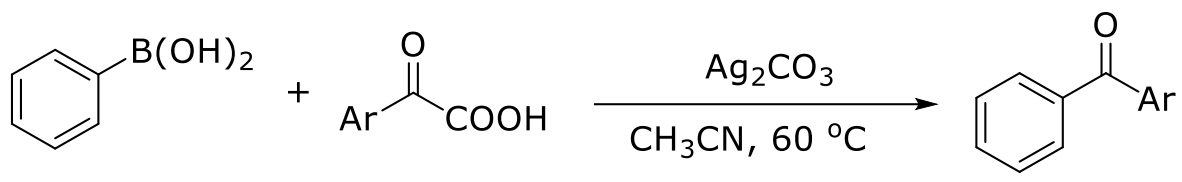

Scheme 5: Silver-catalyzed decarboxylative acylation of 2-oxo-2-phenylacetic acids with arylboronic acids.

The silver reacts with the arylglyoxylic acids derivative (2-oxo-2-phenylacetic acid) to give the corresponding silver salt. Then, decarboxylation occurs, acyl radical is generated in situ. Acyl radical attacks at the phenylboronic acid derivatives to obtain bis-aryl ketones (benzophenone derivatives) in good yields. After the product is obtained, the boronic acid radical $\left[\mathrm{BH}(\mathrm{OH})_{2}\right]$ reacts with silver radical and forms the boric acid and silver cation to complete/restart the cycle. When the radical trapping reagents, such as TEMPO got added into the reaction under the standard conditions independently, no desired product was detected, suggesting that a free radical course was involved in the reaction (Scheme 6). 


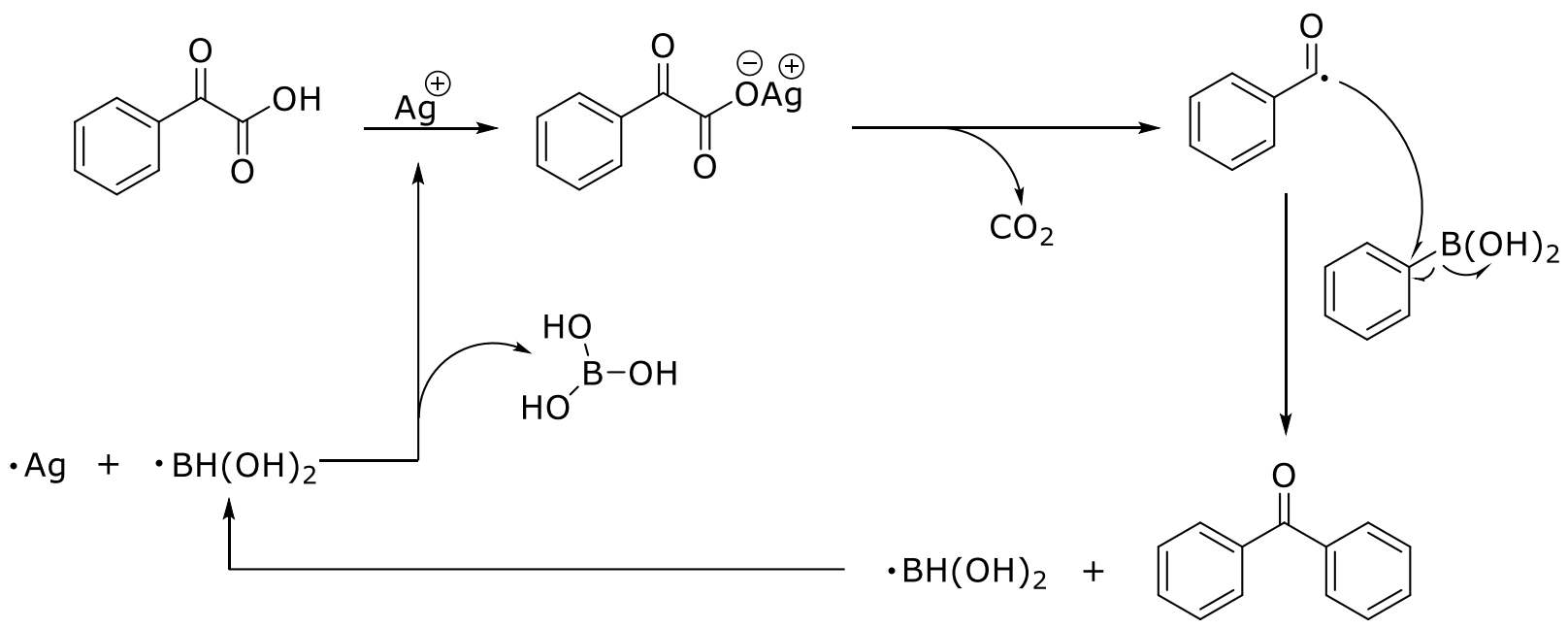

Scheme 6: The proposed mechanism of silver-catalyzed decarboxylative acylation of 2-oxo-2phenylacetic acids with arylboronic acids.

The oxidation of alcohols into the aldehydes or ketones is one of the major and most significant reactions in terms of practical applications in the organic chemistry. In the same year, 2014, Nakazawa and co-workers, by using dehydrogenative reactions, synthesized the derivatives of 2pyridylmethanol via iron complexes (30). Some series of acyl pyridine derivatives were successfully synthesized (Scheme 7).
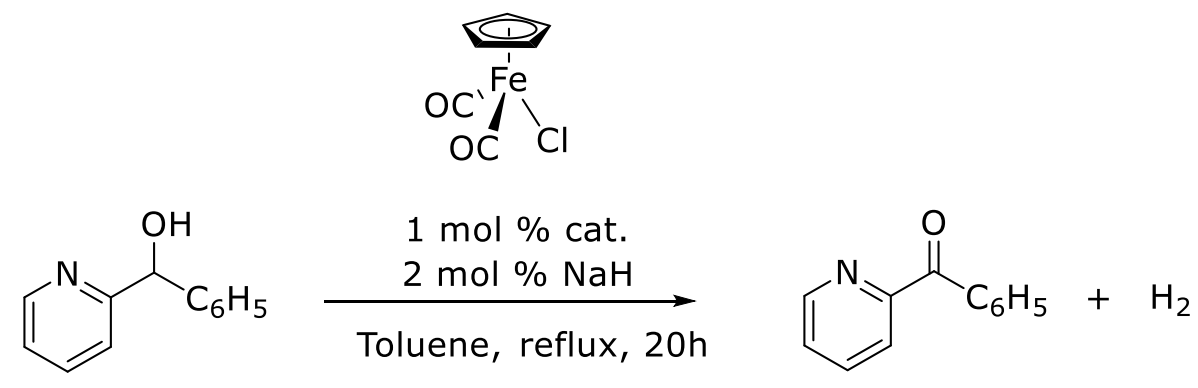

Scheme 7: The dehydrogenation of pyridin-2-ylmethanol via iron complex.

The alcohol reacts with the co-catalyst $\mathrm{NaH}$ to give the corresponding sodium alkoxide. Then this alkoxide reacts with iron complex to give the iron alkoxide complex. Following that, $\mathrm{CO}$ elimination occurs and the nitrogen atom in the attached pyridyl moiety displaces one of the CO ligands to give Intermediate (I). Dissociation of the intermediate (I) takes place, and the subsequent beta-hydride elimination produces the iron hydride complex (II) and the aldehyde product (2pyridinecarboxyaldehyde). The nitrogen atom in the 2-pyridyl group within the first reactant is important for the iron catalyzed dehydrogenation since, 2-thiophenylmethanol (replacing nitrogen with sulfur) did not undergo dehydrogenation and 2-dimethylaminoethanol also did not undergo 
dehydrogenation, indicating that the nitrogen atom in the aromatic ring (2-pyridyl) is important due to steric and/or electronic reasons. This vindicates the fact that nitrogen should be in the aromatic ring for this mechanism to work (Scheme 8).

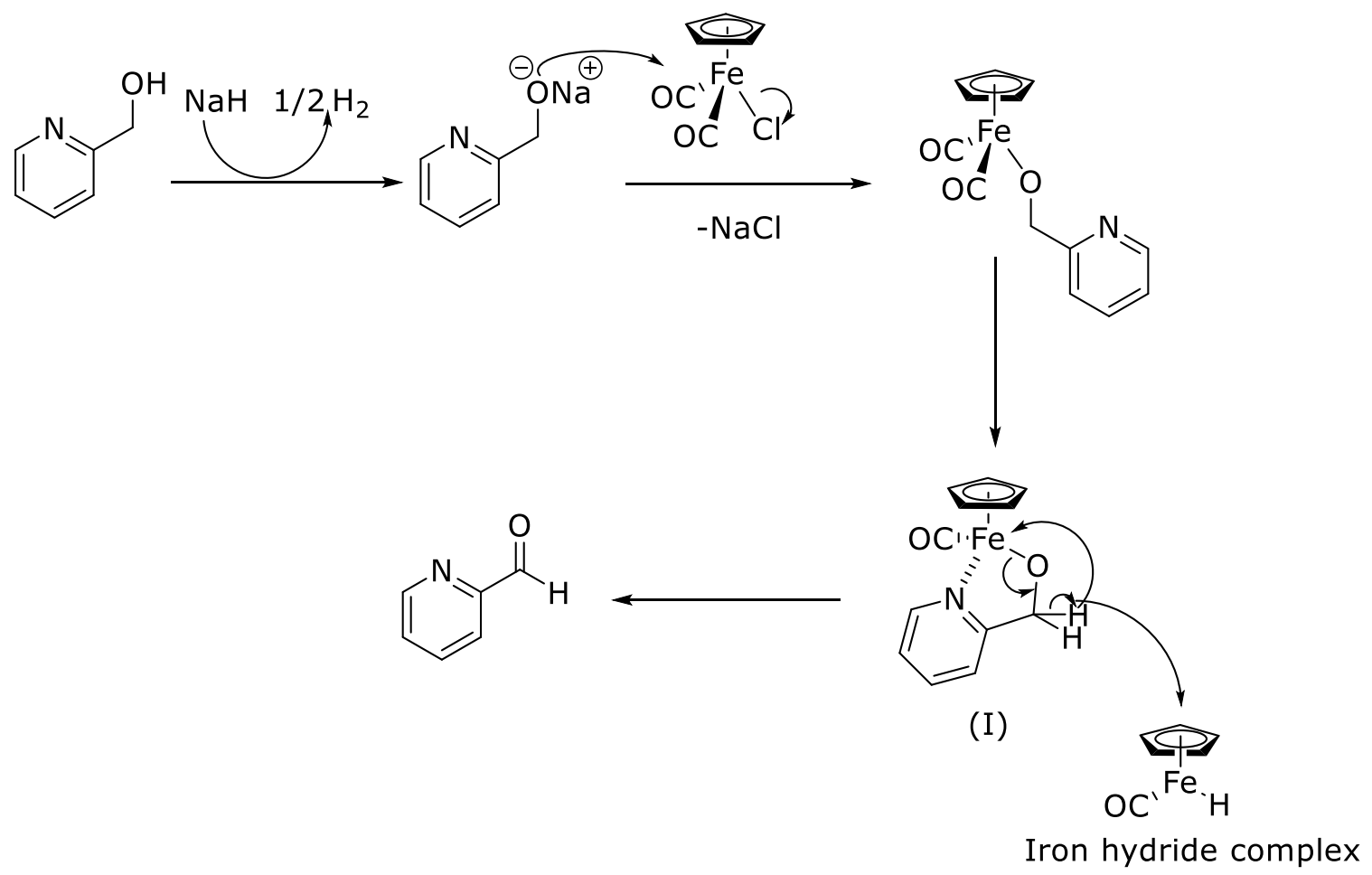

(II)

Scheme 8: The proposed mechanism of dehydrogenation of pyridin-2-ylmethanol via iron complex.

\section{The Metal-Free Acylation and Coupling Reactions}

Gaunt and co-workers successfully synthesized bis-heteroaryl ketones beginning from heteroaryl aldehydes via $\mathrm{N}$-heterocyclic carbene (NHC) catalyst in 2013 (31). Diaryl ketones can both be synthesized via Friedel Crafts reactions or the classical methodology of the addition of carbonyl compounds into the aryl-metal complexes. However, these mentioned synthetic pathways does not work with heteroaryl aldehydes since the organometallic reagents do not fit together with nucleophiles, and also Friedel-Crafts acylation requires n-electron-rich arenes to work better. That is the reason why the synthesis of bis-heteroaryl ketones via NHC catalyst beginning from heteroaryl aldehyde reactants is quite significant (Scheme 9). 


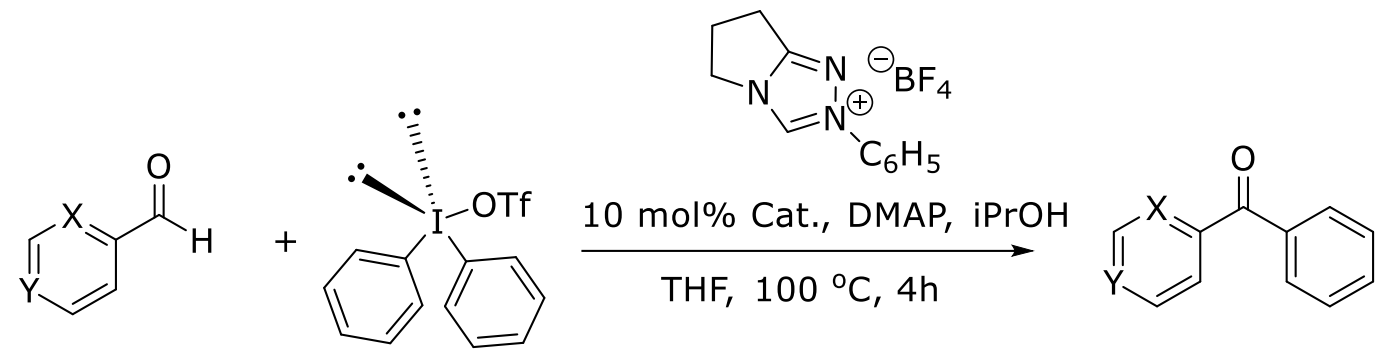

Scheme 9: The proposed mechanism of $N$-heterocyclic carbene-dependent bis-heteroaryl ketone formation via carbogenic nucleophilic transformation.

In this transformation, an NHC catalyst reacts with an aldehyde to form a transient carbogenic nucleophile, Breslow Intermediate (I) with a double bond. This Breslow intermediate's double bond is active to do a nucleophilic attack due to the reason that the nitrogen contributes to the ring aromaticity and the $\Pi$ electrons in the double bond easily attacks at the iodine. It should not be forgotten that triflate is a good leaving group. In the case of intermediate (II), the intermolecular rearrangement occurs and iodine leaves and following that in the case of intermediate (III), nitrogen draws electrons, "nitrogen-heterocyclic carbene" catalyst gets eliminated and the desired product bis-heteroaryl ketone is obtained. The catalytic cycle is now complete (Scheme 10).

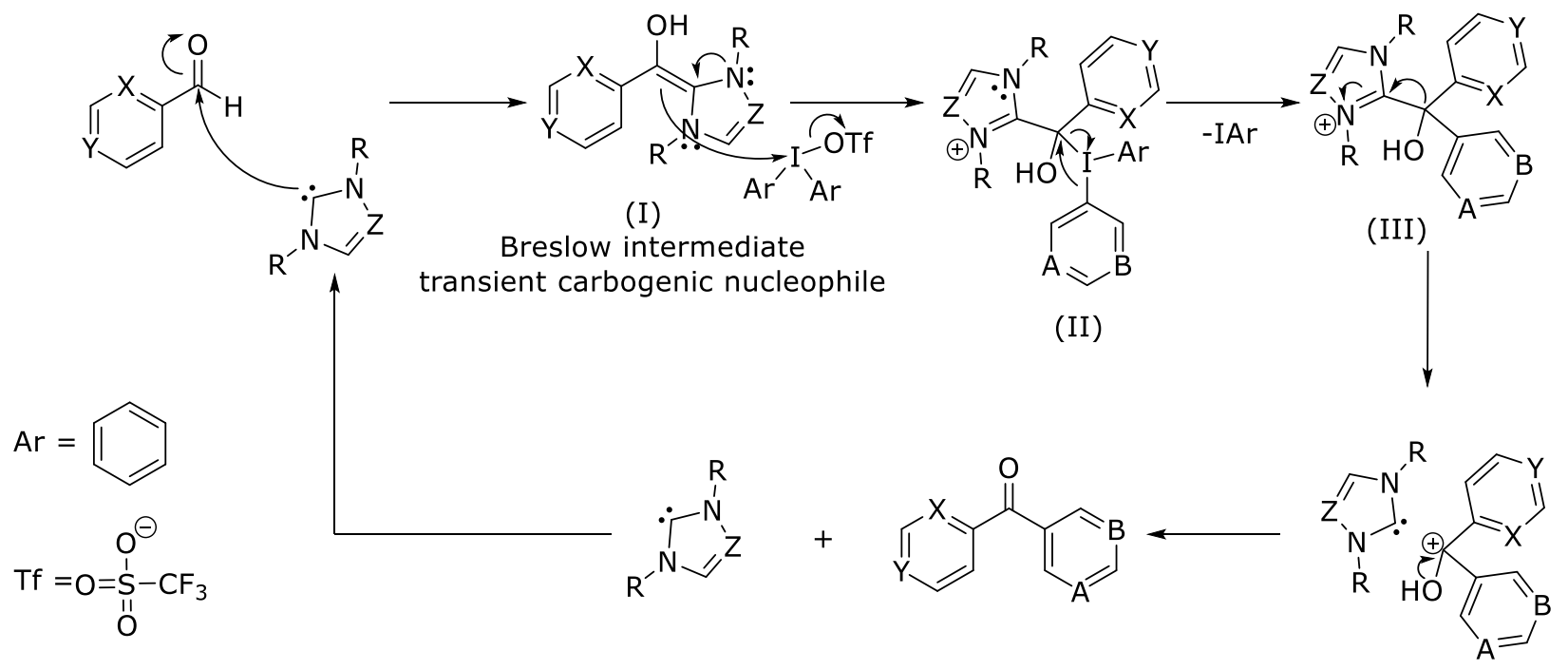

Scheme 10: The proposed mechanism of $N$-heterocyclic carbene dependent bis-heteroaryl ketone formation via carbogenic nucleophilic transformation. 
With respect to a study of Zhaorigetu and co-workers in 2016 with the topic of "Replacing Pd(OAc) 2 with the supported palladium nanoparticles in ortho-directed CDC reactions of alkylbenzenes", the CDC reaction was achieved with the use of palladium nanoparticles instead of using palladium acetate. The "ortho-directing CDC reactions of alkylbenzenes" were possible to be achieved with the use of palladium nanoparticles since, the "supported palladium nanoparticle catalyzed orthodirecting $\mathrm{C}-\mathrm{C}$ coupling reactions of 2-arylpyridines and aldehydes" were stated to be occurring without any repeatability problem. To express and prove how high efficiency and repeatability they possess, it can be said that "Pd/ $\mathrm{y}-\mathrm{Al}_{2} \mathrm{O}_{3}$ catalyst with palladium nanoparticles with the mean diameter sizes of $3.21 \mathrm{~nm}^{\prime \prime}$ showed quite a high catalytic activity and even the best one, compared to the other series of supported palladium nanoparticles. On top of that, without losing any significant catalytic activity, $\mathrm{Pd} / \mathrm{Y}-\mathrm{Al}_{2} \mathrm{O}_{3}$ catalyst with palladium nanoparticles were able to be used five times.

To sum up the study, one can claim that the metallic state palladium is able to catalyze "orthodirecting CDC reaction of alkylbenzenes" including methyl, ethyl and propyl benzene variations for the synthesis of aromatic ketones and palladium states alter within the catalytic cycle. If someone decides to use high catalytic content in an organic reaction, it had been better to choose PdNP catalyst over $\mathrm{Pd}(\mathrm{OAc})_{2}$ since the PdNP catalyst has some beneficial advantages such as higher catalytic activity, easy separation and recycling due to being a heterogeneous catalyst (Scheme 11) (32).
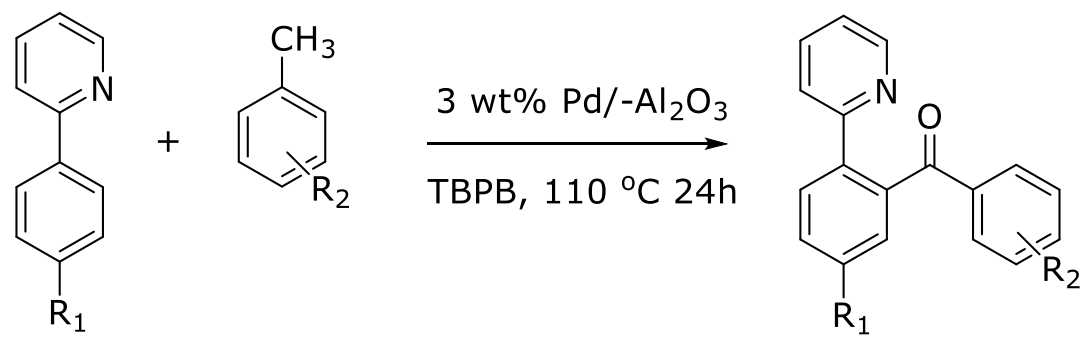

Scheme 11: The synthesis of aromatic ketones via $\mathrm{C}-\mathrm{H}$ functionalization.

\section{The Metal-Free Acylation Reactions via Cross-Dehydrogenative Coupling}

Cross-dehydrogenative coupling (CDC) reactions have developed in time as some quite important tools for the synthesis of varying useful organic compounds due to their atom-economically shorter routes. In general, cross coupling reactions have been quite famous since the beginning of the 2000s depending on the fact that in the $\mathrm{C}-\mathrm{C}$ bond constructions, they possess high regioselectivity and yields, and are especially quite efficient in the construction of varying complex architectures of the ubiquitous building blocks that can be mostly found in natural products. In order to improve new methodologies concerning much greener chemistry with high yields, $\mathrm{C}-\mathrm{H}$ functionalization was 
mostly emphasized nowadays and some quite progress has been observed in $\mathrm{C}-\mathrm{H}$ bond activation in arenes. Within the last decade, these brand new discoveries have led to the achievement of $\mathrm{C}-\mathrm{H}$ bond activation and $\mathrm{C}-\mathrm{C}$ bond formation, solely emerging from two unfunctionalized structures possessing two different $\mathrm{C}-\mathrm{H}$ bonds (33).

One of the main discoveries regarding this field has arrived in the year of 2015. Liu and co-workers published the oxidative dehydrogenative cross-coupling reaction of aldehydes along with the $N$ heterocyclic compounds (34). In their study, they successfully achieved the acylation of isoquinoline derivatives with varying aldehydes. As the oxidant, tert-butyl hydroperoxide (TBHP) and as the acid source trifluoroacetic acid (TFA) were used. TBHP/TFA was chosen due to the fact that in the oxidative cross-dehydrogenative coupling reactions of some varying $N$-heterocycles with diverse aldehydes (under metal free conditions), TBHP/TFA exhibited practical, scalable and highly efficient oxidation medium. These advantages results in the facile synthesis of broad range of structurally diverse C1-acyl substituted heterocycles since, the scope of these varying aldehyde components is broad and these aldehyde components, concerning both aromatic and aliphatic compounds, tolerate electronically varied substituents quite well (Scheme 12).
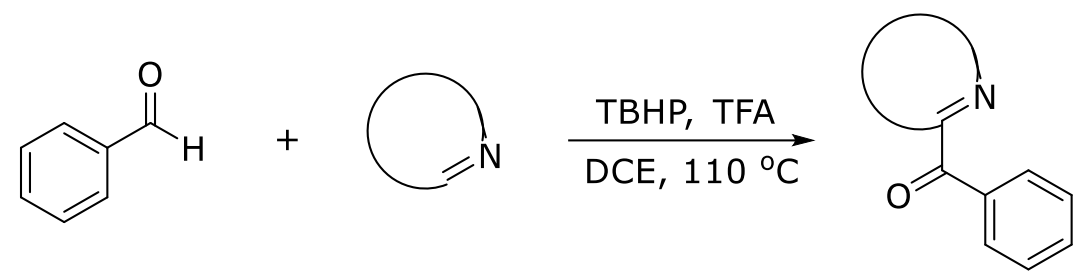

Scheme 12: TBHP/TFA mediated oxidative cross-dehydrogenative coupling of $N$-heterocycles with aldehydes.

Benzaldehyde is converted into its acyl radical in the presence of tert-butylhydroperoxide. "Nitrogen Heteroaryl" (quinoline derivatives) can be activated with TFA to facilitate the addition of intermediate (I) radical onto the "carbon-2 of protonated nitrogen heteroaryl" providing the radical cation intermediate (II). Then this radical cation reacts with the tert-butoxyl radical to yield the desired "acylated quinoline derivatives". When TEMPO is added, no product could be obtained stating that this pathway was in free radical nature (Scheme 13). 


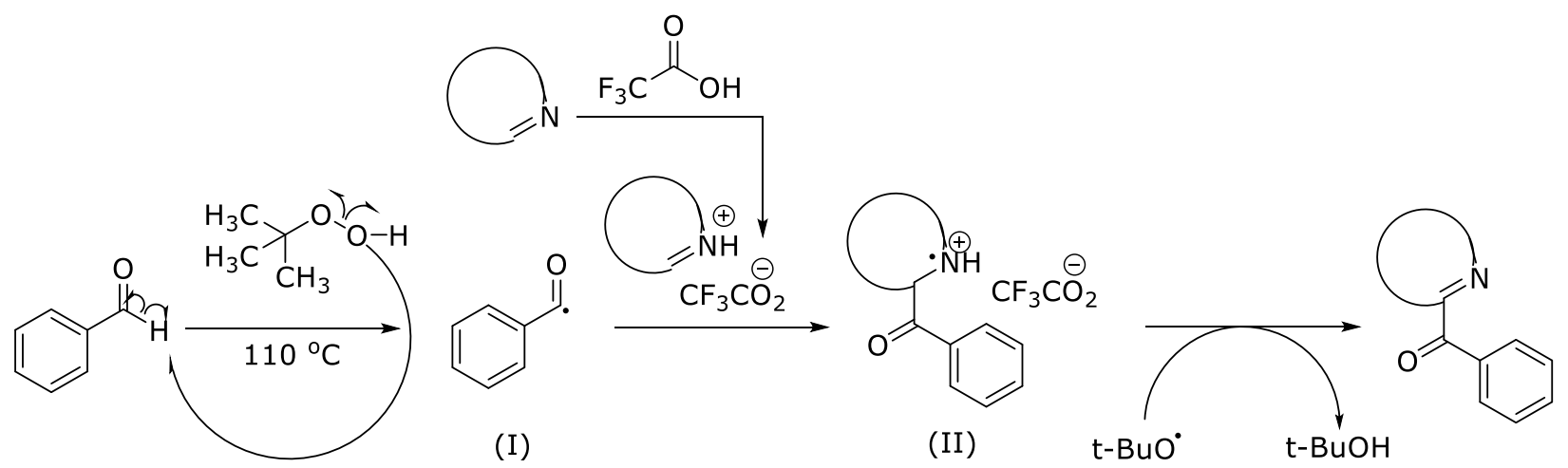

Scheme 13: The proposed mechanism of TBHP/TFA mediated oxidative cross-dehydrogenative coupling of $N$-heterocycles with aldehydes.

Most recently, the acylation of coumarins via CDC approach using aldehydes to serve as acylating agents was investigated in detail by Adib group (35), Qu (36) and Zhou (37). On top of these studies, Duan and co-workers illustrated the Ag-catalyzed diacylation of coumarins using oxocarboxylic acids as the acyl sources (38). In 2016, Adib and co-workers explained an efficient route for the intermolecular double $\mathrm{Csp}^{2}-\mathrm{H}$ functionalization between aromatic aldehydes and 3acetylcoumarins through $\mathrm{Csp}^{2}-\mathrm{Csp}^{2}$ bond formation leading to 4-aroylcoumarin derivatives (39) (Scheme 14).<smiles>[R]C(=O)c1cc2ccccc2oc1=O</smiles>

$$
\underset{\mathrm{CH}_{3} \mathrm{CN}, 80^{\circ} \mathrm{C}, 2-3 \mathrm{~h}}{\stackrel{\mathrm{K}_{2} \mathrm{~S}_{2} \mathrm{O}_{8} \text { Aliquat } 336}{\longrightarrow}}
$$<smiles>[R]C(=O)c1c(C(=O)Br)c2ccccc2oc1=O</smiles>

Scheme 14: The acylation of coumarins via CDC approach.

In order to vindicate the mechanism behind this theory, 4-methylbenzaldehyde and 3acetylcoumarin were chosen as the model reaction substrates to optimize the CDC reaction conditions. In this optimization, the aim was to investigate the effect of several oxidants, solvents, additives, reaction temperature and time as well as various equivalents of oxidants and additives. Initially, this model reaction was tested in the presence of $\mathrm{K}_{2} \mathrm{~S}_{2} \mathrm{O}_{8}$ (1.0 equiv.) as the oxidant and Aliquat 336, tricaprylmethylammonium chloride, (40-42) (15 mol\%) as the additive in acetonitrile at room temperature or $50^{\circ} \mathrm{C}$ for $2 \mathrm{~h}$, but no product was formed. By rising the temperature to a higher degree like $80^{\circ} \mathrm{C}$, 4-arylcoumarin was obtained with $65 \%$ yield. Subsequently, different quantities of $\mathrm{K}_{2} \mathrm{~S}_{2} \mathrm{O}_{8}$ and Aliquat 336 were utilized to improve the yield percentages. Increasing the amount of the additive Aliquat 336 to 30 mole percent led to the desired product 4-arylcoumarin to reach $78 \%$ yield. Ironically, increasing the amount of Aliquat 336 a bit more to 40 mole percent had no effect on the yield. Therefore, another parameter should be altered to see whether any further 
effect on yield can occur or not. Thus, using 1.2 and 1.5 equiv. of $\mathrm{K}_{2} \mathrm{~S}_{2} \mathrm{O}_{8}$ along with 30 mole percent of Aliquat 336 resulted in the desired product 4 -arylcoumarin to be obtained in $87 \%$ and $83 \%$ yields, respectively. Further rising the temperature to $100^{\circ} \mathrm{C}$ decreased the efficiency and yield percentage of the reaction. Some other additives such as NBS, I2, KI and CuI were also screened in the study. However, they had no observable effect in this CDC reaction. Following these tests, the effects on efficiency of varying solvents were carried out with toluene, chlorobenzene, 1,4-dioxane, DCE, DMSO/ $\mathrm{H}_{2} \mathrm{O}(1: 1, \mathrm{v} / \mathrm{v})$, DMSO and $\mathrm{H}_{2} \mathrm{O}$. However, carrying out the reaction in these solvents resulted in the 40-65\% yields of 4-arylcoumarin and in DCE the product was not detected at all. Also, the reaction was performed in the solvent mixture of $\mathrm{CH}_{3} \mathrm{CN} / \mathrm{H}_{2} \mathrm{O}(1: 1, \mathrm{v} / \mathrm{v})$, and the yield was $82 \%$. More testing was also done to indicate the fact that with the use of $\left(\mathrm{NH}_{4}\right)_{2} \mathrm{~S}_{2} \mathrm{O}_{8}$ and TBHP as the oxidants, the yield of 4 -arylcoumarin decreased to $60 \%$ and $40 \%$, respectively. Furthermore, oxidants such as $\mathrm{H}_{2} \mathrm{O}_{2}$ and $D D Q$ were also inert in this $\mathrm{CDC}$ reaction. Without $\mathrm{K}_{2} \mathrm{~S}_{2} \mathrm{O}_{8}$ as the oxidant and Aliquat 336 as the additive, the completion of CDC reaction was not possible to proceed in $\mathrm{CH}_{3} \mathrm{CN}$ at $80^{\circ} \mathrm{C}$ after $2 \mathrm{~h}$. So, the optimal conditions for the metal-free CDC acylation of cumarins were determined as the use of $\mathrm{K}_{2} \mathrm{~S}_{2} \mathrm{O}_{8}$ ( 1.2 equiv.), Aliquat 336 (30 mole percent), acetonitrile as the solvent, $80^{\circ} \mathrm{C}$ as the medium temperature and the duration is 2 hours. To understand and verify the mechanism of $\mathrm{K}_{2} \mathrm{~S}_{2} \mathrm{O}_{8} /$ Aliquat 336 promoted acylation, the reaction between benzaldehyde and 3-acetylcoumarin was performed in the presence of a radical scavenger, 2,2,6,6tetramethylpiperidin-1-yl-oxyl (TEMPO). 2,2,6,6-tetramethylpiperidino benzoate was obtained with $96 \%$ yield and the desired acylated product was not detected at all, proving that an acyl radical was involved in CDC catalytic cycle.

The whole mechanisms described earlier within the review for the use of acylation reactions of simple aromatic compounds and/or aromatic heterocyclic compounds tend to have low/moderate yields and only in some rare cases, have high yields and are quite far away from the green chemistry. However, if such an organic design would be made to make it greener in terms of no use of metal catalysts and if and only if there would a possibility to reach quite high yields without the cost of precision, then it would have become a more feasible, efficient and a compatible design and discovery within the boundaries of green chemistry. Therefore, if there would be such a study, researching a new organic pathway regarding the metal free CDC acylation of heterocyclic compounds such as pyridine derivatives, it would have been a huge contribution to the shelves of organic chemistry library. In that sense, a study with the name of "Metal-free chemo- and regioselective acylation of pyridine derivatives with alcohols in water" was published in 2017 (33). In that study, Kianmehr et al. described a new acylation methodology of pyridine derivatives without using metal catalysts. Beginning the synthesis from benzylic and aliphatic alcohols and using CDC reactions without metal catalysts in water medium, they were able to synthesize 2-acylpyridines for the first time with such a protocol and with such quite high yields as it is shown in (Schemes 15- 
19). The reaction is compatible with accessible aliphatic alcohols and aromatic alcohols as the acylation sources and provides a new route to both alkanoyl- and aroylpyridines. In the general outline of the protocol shown in (Scheme 15), as a radical agent peroxydisulfate $\left(\mathrm{K}_{2} \mathrm{~S}_{2} \mathrm{O}_{8}\right)$ was used. To prove the role of the peroxydisulfate as a radical agent in their mechanism, a control reaction was performed. When the reaction of pyridine was performed in the presence of 2,6-ditert-butyl-4-methylphenol (BHT) as a radical-scavenger, no desired product was obtained.

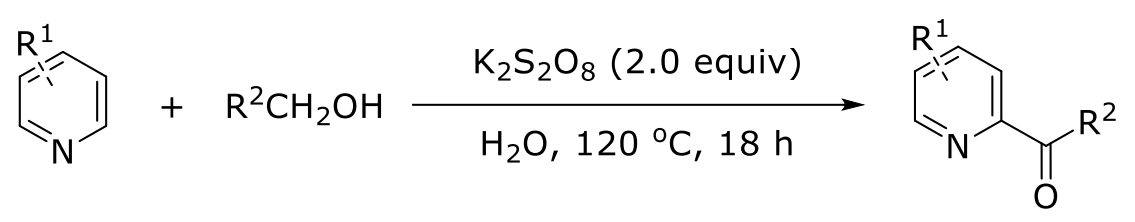

Scheme 15: The synthesis of 2-acylpyridine derivatives.

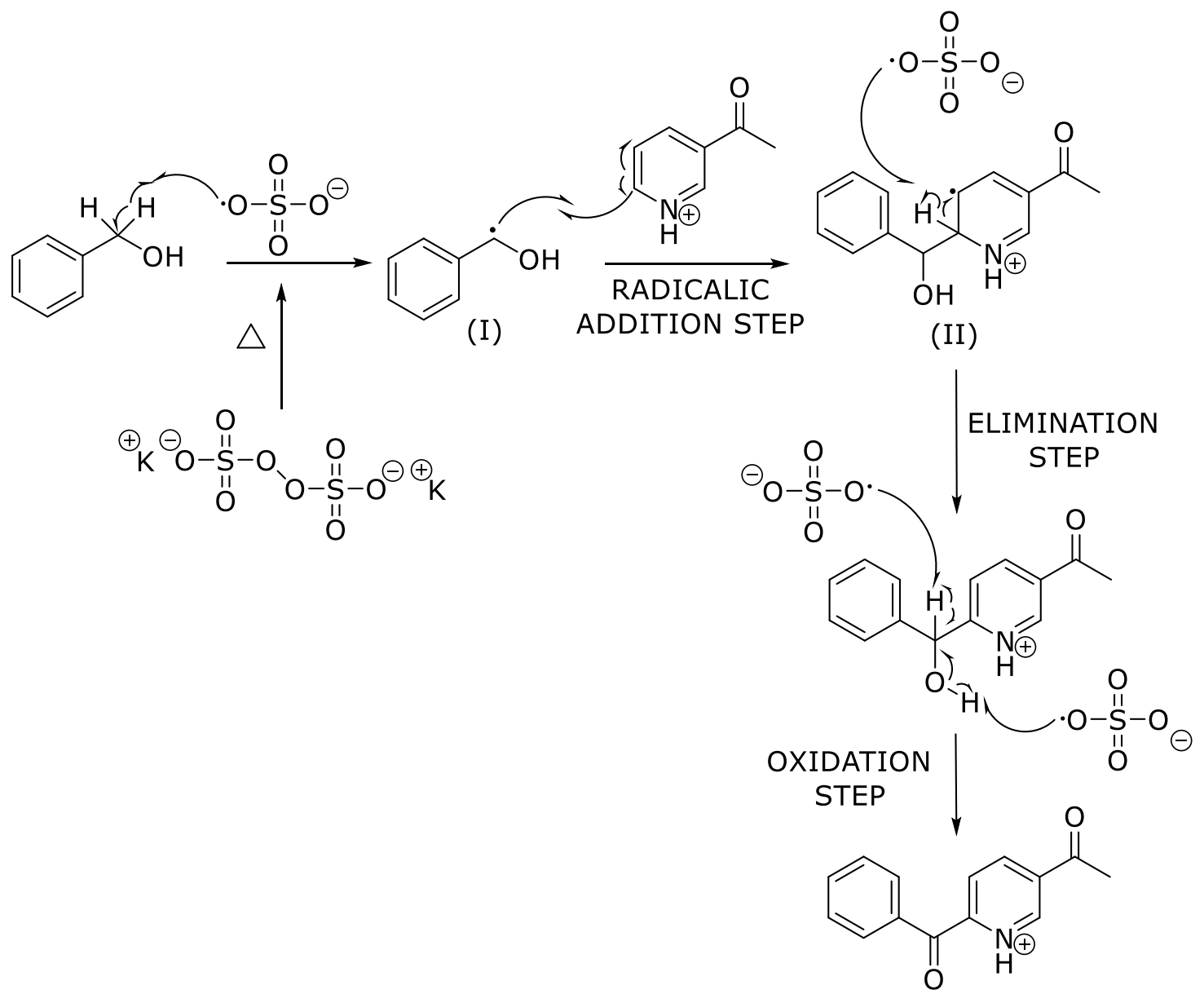

Scheme 16: The first proposed mechanism for the reaction.

In (Scheme 16), intermediate (I) is generated, in situ, through hydrogen atom abstraction from the benzyl alcohol in the presence of sulfate radical anion which is produced from peroxydisulfate $\left(\mathrm{K}_{2} \mathrm{~S}_{2} \mathrm{O}_{8}\right)$. Radical addition of intermediate (I) to pyridine derivative occurs which gets protonated 
under the reaction conditions, giving intermediate (II) which also leads to the formation of the product by elimination followed by oxidation (Medium: $\mathrm{H}_{2} \mathrm{O}$ ).

It should be further discussed that a second pathway (Scheme 17) is also possible for this acylation reaction using benzyl alcohols as the acylation sources. Benzyl alcohol is oxidized to benzaldehyde under the reaction conditions. Hydrogen atom abstraction from benzaldehyde in the presence of sulfate radical anion gives intermediate (III). The obtained free radical attacks the C-2 position of 3-acetylpyridine which is protonated under the reaction conditions, producing the corresponding free radical intermediate (IV). Finally, a hydrogen atom abstraction from the intermediate (IV) yields the desired product.

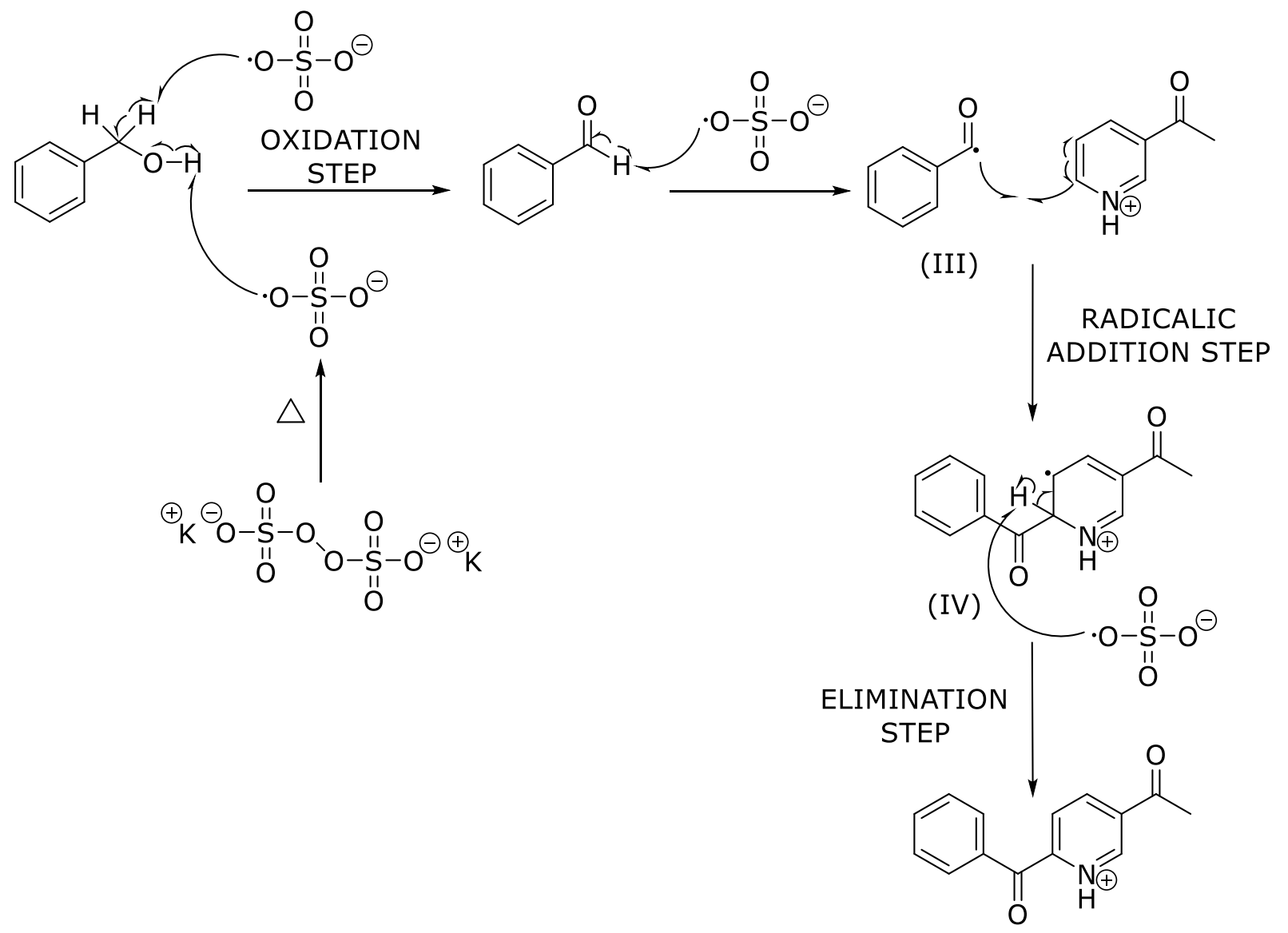

Scheme 17: The second proposed mechanism for the reaction.

This second pathway is confirmed by the observations with a test reaction concerning benzyl alcohol getting oxidized to benzaldehyde in the absence of pyridines under the reaction conditions and also with the fact that, the reaction proceeds successfully with benzaldehydes as the coupling partners instead of benzyl alcohols. 
Agar and Gunkara, JOTCSA. 2018; 5(1): 247-268.

Why there are two proposed mechanisms in which neither of them can be ruled out, is a pretty good question that needs to be answered. If the 2nd pathway yields a product from benzaldehyde, then how can we make sure that the first pathway still works?

Reaction 1

$\stackrel{\mathrm{K}_{2} \mathrm{~S}_{2} \mathrm{O}_{8}(2.0 \text { equiv })}{\mathrm{H}_{2} \mathrm{O}, 120^{\circ} \mathrm{C}, 18 \mathrm{~h}}$

Reaction 2

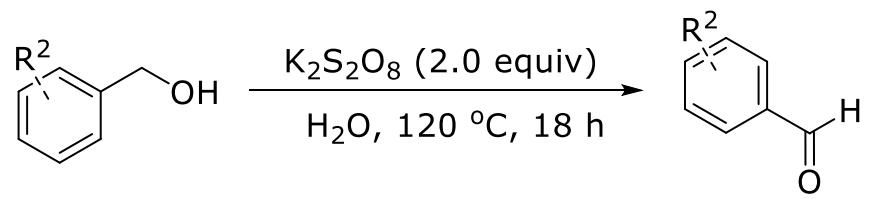

Reaction 3

经

Reaction 4

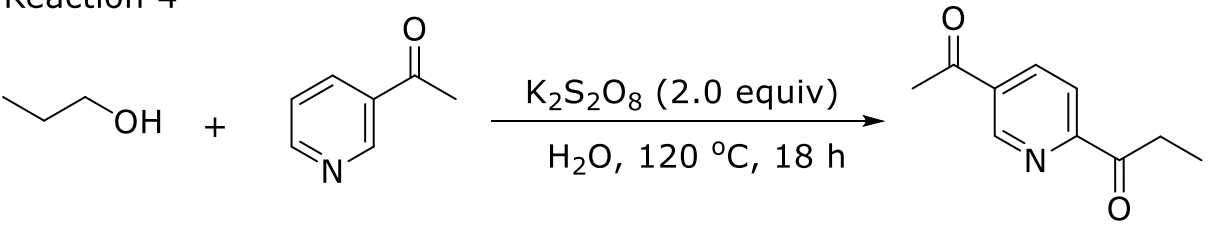

Reaction 5

$\curvearrowright_{\mathrm{OH}} \underset{\mathrm{H}_{2} \mathrm{O}, 120^{\circ} \mathrm{C}, 18 \mathrm{~h}}{\stackrel{\mathrm{K}_{2} \mathrm{~S}_{2} \mathrm{O}_{8}}{\times}(2.0 \text { equiv })} \sim_{\mathrm{H}}^{\mathrm{O}}$

Reaction 6

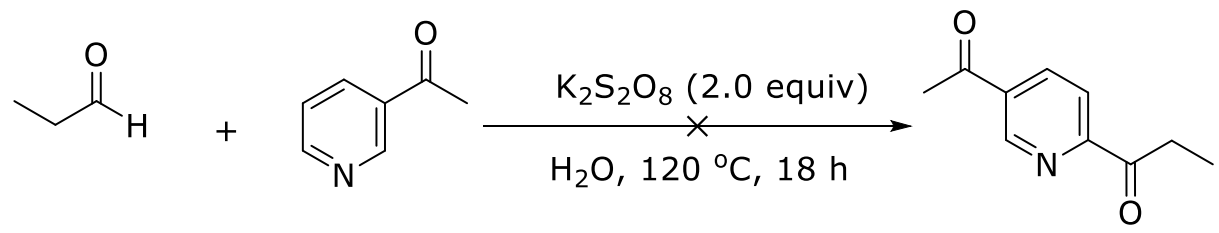

Scheme 18: The General Scheme of all test reactions. 
Agar and Gunkara, JOTCSA. 2018; 5(1): 247-268.

RESEARCH ARTICLE

Regarding this issue, (Scheme 18) will be helpful to comprehend the big picture behind it. In reaction 1 , benzyl alcohol reacts with pyridine derivative yielding the final desired products, proving that the total reaction works. When it is taken a look at the reaction 2 , the second proposed pathway is confirmed by the observation that was done where benzyl alcohol is oxidized to the corresponding aldehyde in the absence of pyridine (under the same reaction conditions). In the case of reaction 3 , beginning the synthesis from the aldehyde immediately vindicates the second proposed pathway since it yields the final product. In reaction 4, when the aliphatic alcohol reacts with pyridine derivative, it yields the final product, showing that the total reaction works for aliphatics as well. In reaction 5, when it was again tried with the aliphatic alcohol but this time without pyridine, it was observed that the oxidation of ethanol and propanol to the corresponding aldehydes was not successful. This incident supports the first proposed mechanism. Therefore, any idea that may come into the mind to rule out the first mechanism cannot be possible after the incident of this test reaction. In the final reaction trial, the sixth one simply states that the total reaction failed to proceed when it was started from aliphatic aldehydes (ethanal and propanal) as the coupling partners where this again supports the first proposed mechanism.

The result that can be obtained from these test reactions is that the mechanisms concerning benzyl alcohol can both include the first and second proposed mechanisms. However, in the case of aliphatic alcohols, solely the first mechanism works. In the case of where benzyl alcohol is the reactant, benzylic radical due to its own aromaticity is more stable than the aliphatic alcohol in terms of getting attacked by a radical. Therefore, the second proposed mechanism's first oxidation step cannot occur when the aliphatic alcohol is the reactant.

All in all, Kianmehr et al. efficiently designed organic pathways which are practical methodologies for the chemoselective and regioselective synthesis of 2-acylpyridines, beginning from the pyridine and some varying aliphatic and benzylic alcohol reactants. Besides the good yields that can be seen in (Scheme 19), throughout the products of 6 to 9, the yields are moderately good when electron withdrawing groups such as cyanide, acetyl, ester are attached to the pyridine ring. Besides that, one of the main adventageous sides of this study is that it was performed within the water medium. Thus, this leads to the fact that it can be applied to decipher new materialistic discoveries especially in biotechnology and material science/engineering since the discovered routes to synthesize 2acylpyridines are pretty straightforward, possess high yields, requires environmentally compatible and mild medium such as the use of water as a solvent and the benefit of not being forced to use any metal catalysts. Hence, if some new materials and compounds possessing good tolerance of various functional groups gets desired to be studied in the future, these kind of new acylation pathways just like the ones in this study, are always helpful for new technological advancements. 
<smiles>CC(=O)c1ccc(C(=O)c2ccccc2)nc1</smiles>

$1(87 \%)$<smiles>CC(C)(C)c1ccc(C(=O)c2ccccn2)cc1</smiles>

$4(71 \%)$<smiles>CC(=O)c1ccnc(C(=O)c2ccc(C)cc2)c1</smiles>

$7(82 \%)$<smiles>CCC(=O)c1ccc(C(C)=O)cn1</smiles>

$10(85 \%)$<smiles>CC(=O)c1ccc(C(=O)c2ccc(C)cc2)nc1</smiles>

$2(85 \%)$<smiles>O=C(c1ccccc1)c1ccccn1</smiles>

$5(85 \%)$<smiles>CCOC(=O)c1ccc(C(=O)c2ccccc2)nc1</smiles>

$8(66 \%)$<smiles>CCCCC(=O)c1ccc(C(C)=O)cn1</smiles>

$11(86 \%)$<smiles>CC(=O)c1ccc(C(=O)c2ccc(Cl)cc2)nc1</smiles>

$3(83 \%)$<smiles>Cc1ccc(C(=O)c2cc(C#N)ccn2)cc1</smiles>

$6(68 \%)$<smiles>CC(=O)c1ccc(C(C)=O)nc1</smiles>

$9(89 \%)$<smiles>CC(=O)c1ccc(C(=O)CC(C)C)nc1</smiles>

$12(69 \%)$

Scheme 19: Various products of 2-acylpyridines.

\section{CONCLUSION}

The acylation of aromatic compounds has quite a significant role both within the scientific advancement of organic chemistry itself and its technological applications in which serving as building blocks in the material science/engineering, drug designs, pharmaceuticals and polymer chemistry. Due to the importance of this field, different research institutes throughout the world try to decipher mechanisms concerning varying $\mathrm{CDC}$ reaction types for the use of acylation reaction in the organic chemistry. Thus, as it can be seen from what was mentioned earlier within the review paper, the corner stone mechanisms regarding the metal catalysts and/or metal-free CDC reactions for the use of acylation reactions in the aromatic compounds have been discussed comprehensively so far. Among the examined cross coupling reactions, Kianmehr et al.'s new protocol published in 
Agar and Gunkara, JOTCSA. 2018; 5(1): 247-268.

RESEARCH ARTICLE

2017, represents a more environmentally benign, practical, high yielding and cost effective methodology for the acylation of aromatic compounds.

\section{REFERENCES}

1. Hashimoto I, Kawaji T, Badea FD, Sawada T, Mataka S, Tashiro M, et al. Regioselectivity of Friedel-Crafts acylation of aromatic compounds with several cyclic anhydrides. Res Chem Intermediat. 1996;22(9):855-69.

2. Freese U, Heinrich F, Roessner F. Acylation of aromatic compounds on H-Beta zeolites. Catal Today. $1999 ; 49(1-3): 237-44$.

3. Ma ZH, Zhang XL, Wang H, Han ZG, Zheng XZ, Lin J. Syntheses, structures and catalytic activity for FriedelCrafts reactions of substituted indenyl rhenium carbonyl complexes. J Coord Chem. 2017;70(4):709-21.

4. Yeung CS, Dong VM. Catalytic Dehydrogenative Cross-Coupling: Forming Carbon-Carbon Bonds by Oxidizing Two Carbon-Hydrogen Bonds. Chem Rev 2011;111(3):1215-1292.

5. Beccalli EM, Broggini G, Martinelli M. Sottocornola S, C-C, C-O, C-N bond formation on $\mathrm{sp}(2)$ carbon by Pd(II)-catalyzed reactions involving oxidant agents. Chem Rev 2007;107(11):5318-5365.

6. Huang H, Stewart T, Gutmann M, Ohhara T, Niimura N, Li YX, Wen JF, Bau R, Wong HNC. To Flip or Not To Flip? Assessing the Inversion Barrier of the Tetraphenylene Framework with Enantiopure 2,15Dideuteriotetraphenylene and 2,7-Dimethyltetraphenylene. J Org Chem 2009;74(1):359-369.

7. Ling CMS, Keita T, Qinghao C, Natalya P, Eric S, Jin-Quan Y. Remote Meta-C-H Activation Using a PyridineBased Template: Achieving Site-Selectivity via the Recognition of Distance and Geometry. ACS Cent. Sci. 2015;1:394-399.

8. Lyons TW, Sanford MS. Palladium-Catalyzed Ligand-Directed C-H Functionalization Reactions. Chem Rev 2010;110(2):1147-1169.

9. Li ZP, Li CJ. Catalytic allylic alkylation via the cross-dehydrogenative-coupling reaction between allylic $\mathrm{sp}(3)$ $\mathrm{C}-\mathrm{H}$ and methylenic sp(3) C-H bonds. J Am Chem Soc 2006;128(1):56-57.

10. Li XL, Hewgley JB, Mulrooney CA, Yang JM, Kozlowski MC. Enantioselective oxidative biaryl coupling reactions catalyzed by 1,5-diazadecalin metal complexes: Efficient formation of chiral functionalized BINOL derivatives. J Org Chem 2003;68(14):5500-5511.

11. Guo XW, Pan SG, Liu JH, Li ZP. One-Pot Synthesis of Symmetric and Unsymmetric 1,1-Bis-indolylmethanes via Tandem Iron-Catalyzed C-H Bond Oxidation and C-O Bond Cleavage. J Org Chem 2009;74(22):8848-8851.

12. Li CJ, Li Z. Green chemistry: The development of cross-dehydrogenative coupling (CDC) for chemical synthesis. Pure Appl. Chem. 2006;78:935-945.

13. Li Z, Bohle DS, Li CJ. Cu-catalyzed cross-dehydrogenative coupling: A versatile strategy for C-C bond formations via the oxidative activation of sp ${ }^{3} \mathrm{C}-\mathrm{H}$ bonds. Proc. Natl. Acad. Sci. U. S. A. 2006;103:8928-8933.

14. Beccalli EM, Broggini G, Martinelli M, Sottocornola S. C-C, C-O, C-N Bond Formation on $\mathrm{sp}^{2}$ Carbon by Pd(II)-Catalyzed Reactions Involving Oxidant Agents. Chem. Rev. 2007;107(11):5318-5365.

15. Li CJ. Cross-Dehydrogenative Coupling (CDC): Exploring $\mathrm{C}-\mathrm{C}$ Bond Formations beyond Functional Group Transformations. Acc. Chem. Res. 2009;42(2):335-344.

16. Scheuermann CJ. Beyond Traditional Cross Couplings: The Scope of the Cross Dehydrogenative Coupling Reaction. Chem. Asian J. 2010;5:436-451.

17. Yoo, WJ, Li CJ. Cross-Dehydrogenative Coupling Reactions of sp3-Hybridized C-H Bonds. Top. Curr. Chem. 2010;292:281-302. 
18. Yeung CS, Dong VM. Catalytic Dehydrogenative Cross-Coupling: Forming Carbon-Carbon Bonds by Oxidizing Two Carbon-Hydrogen Bonds. Chem. Rev. 2011;111(3):1215-1292.

19. Cho SH, Kim JY, Kwak J, Chang S. Recent advances in the transition metal-catalyzed twofold oxidative C$\mathrm{H}$ bond activation strategy for $\mathrm{C}-\mathrm{C}$ and $\mathrm{C}-\mathrm{N}$ bond formation. Chem. Soc. Rev. 2011;40(10):5068-5083.

20. Sun CL, Li BJ, Shi ZJ. Direct C-H Transformation via Iron Catalysis. Chem. Rev. 2011;111(3):1293-1314.

21. Zhang C, Tang C, Jiao N. Recent advances in copper-catalyzed dehydrogenative functionalization via a single electron transfer (SET) process. Chem. Soc. Rev. 2012;41(9):3464-3484.

22. Song G, Wang F, Li X. C-C, C-O and $\mathrm{C}-\mathrm{N}$ bond formation via rhodium(III)-catalyzed oxidative $\mathrm{C}-\mathrm{H}$ activation. Chem. Soc. Rev. 2012;41:3651-3678.

23. Hirano K, Miura M. Copper-mediated oxidative direct C-C (hetero)aromatic cross-coupling. Chem. Commun. 2012;48:10704-10714.

24. Liu C, Liu D, Lei A. Recent Advances of Transition-Metal Catalyzed Radical Oxidative Cross-Couplings. Acc. Chem. Res. 2014;47(12):3459-3470.

25 Girard SA, Knauber T, Li CJ. The Cross-Dehydrogenative Coupling of $\mathrm{C}_{\mathrm{sp} 3}-\mathrm{H}$ Bonds: A Versatile Strategy for C-C Bond Formations. Angew. Chem., Int. Ed. 2014;53:74-100.

26. Samanta R, Matcha K, Antonchick AP. Metal-Free Oxidative Carbon-Heteroatom Bond Formation Through C-H Bond Functionalization. Eur. J. Org. Chem. 2013;26:5769-5804.

27. Tang BX, Song RJ, Wu CY, Liu Y, Zhou MB, Wei WT, Deng G, Yin D, Li J. Copper-Catalyzed Intramolecular $\mathrm{C} \mathrm{H}$ Oxidation/Acylation of Formyl- $\mathrm{N}$-arylformamides Leading to Indoline-2,3-diones. J Am Chem Soc. 2010;132(26):8900-+.

28. Jiang $\mathrm{HL}$, Xie J, Lin AJ, Cheng YX, Zhu CJ. The Au(III)-catalyzed coupling reactions between alcohols and N-heterocycles via C-H bond activation. Rsc Adv. 2012;2(28):10496-8.

29. Cheng K, Zhao BL, Qi CZ. Silver-catalyzed decarboxylative acylation of arylglyoxylic acids with arylboronic acids. Rsc Adv. 2014;4(89):48698-702.

30. Kamitani M, Ito M, Itazaki M, Nakazawa H. Effective dehydrogenation of 2-pyridylmethanol derivatives catalyzed by an iron complex. Chem Commun (Camb). 2014;50(59):7941-4.

31. Toh QY, McNally A, Vera S, Erdmann N, Gaunt MJ. Organocatalytic C-H bond arylation of aldehydes to bisheteroaryl ketones. J Am Chem Soc. 2013;135(10):3772-5.

32. Bao YS, Zhang DL, Jia ML, Bao ZRGT. Replacing $\operatorname{Pd}(O A C)(2)$ with supported palladium nanoparticles in ortho-directed CDC reactions of alkylbenzenes. Green Chem. 2016;18(7):2072-7.

33. Kianmehr E, Pakbaznia A, Faghih N, Forournadi A. Metal-free chemo- and regioselective acylation of pyridine derivatives with alcohols in water. Tetrahedron. 2017;73(11):1407-12.

34. Chen J, Wan M, Hua J, Sun Y, Lv Z, Li W, et al. TBHP/TFA mediated oxidative cross-dehydrogenative coupling of $\mathrm{N}$-heterocycles with aldehydes. Org Biomol Chem. 2015;13(47):11561-6.

35. Adib M, Rajai-Daryasarei S, Pashazadeh R, Tajik M, Mirzaei P. Regioselective transition metal-free acylation of coumarins via cross-dehydrogenative coupling reaction of coumarins and aldehydes. Tetrahedron Lett. $2016 ; 57(33): 3701-3705$.

36. Yuan JW, Yin QY, Yang LR, Mai WP, Mao P, Xiao YM, Qu LB. Iron-catalyzed regioselective direct coupling of aromatic aldehydes with coumarins leading to 3-aroyl coumarins. RSC Adv. 2015; 5: 88258-88265. 
37. Zhao W, Xu L, Ding Y, Niu B, Xie P, Bian Z, Zhang D, Zhou A. Regioselective Coupling Reactions of Coumarins with Aldehydes or Di-tert-butyl Peroxide (DTBP) through a C(sp2)-H Functionalization Process. Eur. J. Org. Chem. 2016;2:325-330.

38. Wang H, Zhou SL, Guo LN, Duan XH. Diacylation of coumarins by silver-catalyzed decarboxylative crosscoupling. Tetrahedron. 2015;71(4):, 630-636.

39. Adib M, Pashazadeh R, Rajai-Daryasarei S, Kabirib R, Jahania M. Transition metal-free crossdehydrogenative coupling acylation of coumarins by the $\mathrm{K}_{2} \mathrm{~S}_{2} \mathrm{O}_{8} /$ Aliquat 336 catalytic system: a versatile strategy towards 4-aroylcoumarin derivatives. RSC Adv. 2016;6: 110656-110660.

40. Starks CM. Phase-transfer catalysis. I. Heterogeneous reactions involving anion transfer by quaternary ammonium and phosphonium salts. J. Am. Chem. Soc. 1971;93(1):195-199.

41. Grudpan K, Taylor CG. Use of Aliquat-336 for the extraction of cadmium from aqueous solutions. Analyst, $1984 ; 109: 585-588$.

42. Mikkola JP, Virtanen P, Sjoholm R. Aliquat $336 \AA-a$ versatile and affordable cation source for an entirely new family of hydrophobic ionic liquids. Green Chem. 2006;8:250-255. 
